\title{
In Silico Characterization of Repolarization Duration and Variability in the Long QT1 Syndrome Under $\beta$-Adrenergic Stimulation
}

\author{
David A Sampedro-Puente ${ }^{1}$, Fabien Raphel ${ }^{2}$, Jesus Fernandez-Bes ${ }^{1}$, Pablo Laguna ${ }^{1}$, Damiano \\ Lombardi $^{2}$, Esther Pueyo ${ }^{1}$ \\ ${ }^{1}$ University of Zaragoza, IIS Aragón \& CIBER-BBN, Zaragoza, Spain \\ 2 INRIA \& LJLL Sorbonne Université, Paris, France
}

\begin{abstract}
Enhanced temporal repolarization variability facilitates ventricular arrhythmias in the long QT 1 (LQT1) syndrome, particularly under $\beta$-adrenergic stimulation $(\beta$ $A S)$. The underlying mechanisms are, however, not fully elucidated. In silico investigation of such mechanisms first requires methods able to reproduce the experimental observations. Here, we describe a method for identification of in silico action potential (AP) models from input voltage traces and we apply it to investigate repolarization variability in LQT1. A combination of Double Greedy Dimension Reduction (DGDR) Unscented Kalman Filter (UKF) was used to estimate the ionic conductances and phosphorylation levels of coupled $A P$ and $\beta$-AS models. Over synthetic AP traces from an experimentally-calibrated population of LQT1 cells, combined DGDR-UKF accurately estimated the model parameters, with reduced estimation uncertainty and convergence time. Importantly, combined $D G D R-U K F$ was able to reliably replicate the statistical distributions of AP duration and short-term variability, both at baseline and under $\beta-A S$, with relative errors below 4\%. Arrhythmogenic AP alternans were reproduced too. In conclusion, our method allows characterization of repolarization duration and variability in LQT1, which is expected to help disentangling the mechanisms underlying adrenergic-induced arrhythmias in this syndrome.
\end{abstract}

\section{Introduction}

Long QT (LQT) syndrome is a congenital disease that causes abnormal QT prolongation and leads to high risk of life-threatening arrhythmias. The most common LQT variant is LQT type 1 (LQT1), in which more than $90 \%$ of lethal events occur during physical or emotional stress, in association with strong sympathetic stimulation [1]. In vivo and in vitro LQT1 studies have reported a link between enhanced spatio-temporal repolarization variability and increased risk of ventricular arrhythmias and sud- den cardiac death, particularly in response to $\beta$-adrenergic stimulation ( $\beta$-AS) [2]. Further investigation into the mechanisms behind such observations and the establishment of robust markers that anticipate arrhythmic risk is still needed, which could be highly relevant for the development of improved therapies for the LQT1 syndrome.

Computational modeling and simulation has become a powerful tool to complement experimental and clinical research, contributing to advance our understanding of cardiac electrophysiology in health and disease. In particular, methodologies for parameter estimation of action potential (AP) computational models from available experimental data has demonstrated great potential towards the development of personalized in silico models. Such models could allow investigating the mechanisms underlying electrophysiological properties and their relation to increased arrhythmic risk, narrowing the number of experiments by guiding them according to simulation predictions. In recent studies, parameter estimation approaches based on Double Greedy Dimension Reduction (DGDR) [3] and the Unscented Kalman Filter (UKF) [4] have been shown to accurately identify the parameters and state variables of stochastic human ventricular AP models $[5,6]$.

In this work, we test the performance of individual and combined UKF and DGDR methods to identify the parameters of coupled electrophysiology and $\beta$-AS computational models from given input voltage AP traces. Based on such identification, we establish the capacity of the methods to reproduce spatio-temporal repolarization variability in LQT1 and investigate arrhythmogenic phenomenal related to enhanced variability.

\section{Material and Methods}

\subsection{Stochastic Population of AP Models}

A population of stochastic AP models with representation of spatio-temporal variability was constructed from a stochastic version of the O'Hara et al. human ventricular model [7] by varying the conductances of eight ionic cur- 
rents in the range $\pm 100 \%$ of their nominal values. The currents were: $I_{K s}, I_{K r}, I_{t o}, I_{C a L}, I_{K 1}, I_{N a}, I_{N a C a}$ and $I_{\mathrm{NaK}}$. From the 8000 models initially generated by a Monte-Carlo method, only 2373 models were retained after a calibration step based on physiological limits of electrical properties, as described in [6]. The calibrated models represent virtual cells with distinct ionic properties.

$\beta$-AS effects were described by the Xie et al. model $[8,9]$, with the updates described in [10]. Here, we considered $\beta$-AS effects to be equal for all cells except for phosphorylation of the three cellular substrates with more remarkable impact on the AP, namely $I_{K s}, I_{C a L}$ and $I_{N a K}$ [10]. Multiplying factors applied to the PKA phosphorylation levels of these three substrates, denoted by $f_{C a L}, f_{K s}$ and $f_{\mathrm{NaK}}$, were varied in ranges spanning from baseline values to values reached after application of a saturating isoproterenol dose of $1 \mu \mathrm{M}$. A Monte-Carlo method was used to generate a population containing variations in such multiplying factors.

By combining the described variations in eight ionic conductances and three phosphorylation levels, a population of 2373 stochastic cell models was constructed that represented both baseline and $\beta$-AS conditions. LQT1 conditions were modeled by $90 \%$ reduction in the $I_{K s}$ current conductance.

\subsection{Parameter Estimation}

For a given input voltage trace, which could eventually be obtained experimentally or from a computational simulation, a method was used to estimate the parameters of an underlying AP model. As described in Section 2.1, these parameters represent ionic conductances and phosphorylation levels of an electrophysiological-adrenergic AP model. The employed approach combines DGDR and UKF methods.

DGDR, a methodology for high-dimensional data analysis [3], was first applied to estimate the AP model parameters by projecting the input data into a low subspace through a sparse linear combination of dictionary entries. The dictionary was composed by different AP markers, like AP duration at different percentages of repolarization, short-term variability (STV) of AP duration, peak voltage, resting membrane potential or wavelet decomposition components, among others, as well as linear combinations of these markers [6].

The parameter estimates obtained by application of DGDR were used to initialize the estimates of a subsequently applied UKF-based method. This UKF method allowed estimation of the AP model parameters by formulating a nonlinear state-space representation and approximating the posterior distributions of the states through propagation of so-called Sigma Points [11]. Additionally, at the end of each cardiac beat, UKF estimates were updated based on the mean and covariance of the DGDR estimates so as to avoid local minima that could lead UKF estimates far from the actual parameter values.

The performance of the combined DGDR-UKF method was tested on the experimentally-calibrated population of models described in Section 2.1 and compared to the performances of the individual DGDR and UKF methods at baseline and in response to $\beta$-AS. For that purpose, the model population was split into a training subpopulation of 2000 virtual cells and a validation subpopulation of 373 virtual cells. In a next step, LQT1 conditions were applied onto the input and estimated populations and these were compared in terms of reproduction of the AP-derived markers described in the next section.

\subsection{AP Markers}

AP-derived markers were calculated from $N$ beats of actual and estimated AP traces and included: APD, calculated as the mean of AP durations at $90 \%$ repolarization $\left(\mathrm{APD}_{90}\right)$ of individual beats, and STV, calculated as the average distance perpendicular to the identity line in the Poincaré plot of $\mathrm{APD}_{90}[5]$ :

$$
\begin{gathered}
\mathrm{APD}=\frac{1}{N} \sum_{n=1}^{N} \operatorname{APD}_{90}(n), \\
S T V=\sum_{n=1}^{N-1} \frac{\left|\operatorname{APD}_{90}(n+1)-\operatorname{APD}_{90}(n)\right|}{(N-1) \sqrt{2}} .
\end{gathered}
$$

\section{Results and Discussion}

\subsection{Repolarization Variability in LQT1}

Fig. 1 shows the Poincaré plots of $\mathrm{APD}_{90}$ for two virtual cells of the model population described in section 2 (models \#5 and \#330) at four different scenarios: baseline, $\beta$-AS, LQT1 and LQT1 with $\beta$-AS. When the voltage traces of each of these cells, both at baseline and under $\beta$-AS, were input to the DGDR, UKF and DGDR-UKF methods described in section 2.2, estimates of ionic conductances and phosphorylation levels were obtained, from which AP traces were generated. The Poincaré plots of estimated $\mathrm{APD}_{90}$ for each of the three tested methods are presented in the same figure. As can be observed, the combined DGDR-UKF method outperformed individual DGDR and UKF in reproducing not only the mean $\mathrm{APD}_{90}$ but also its temporal variability, with DGDR-UKF rendering data points closely matching the input ones. After simulation of LQT1 conditions by $I_{K s}$ inhibition, differences between the tested estimation methods were even more accentuated, with DGDR-UKF reproducing APD and STV with high reliability. 

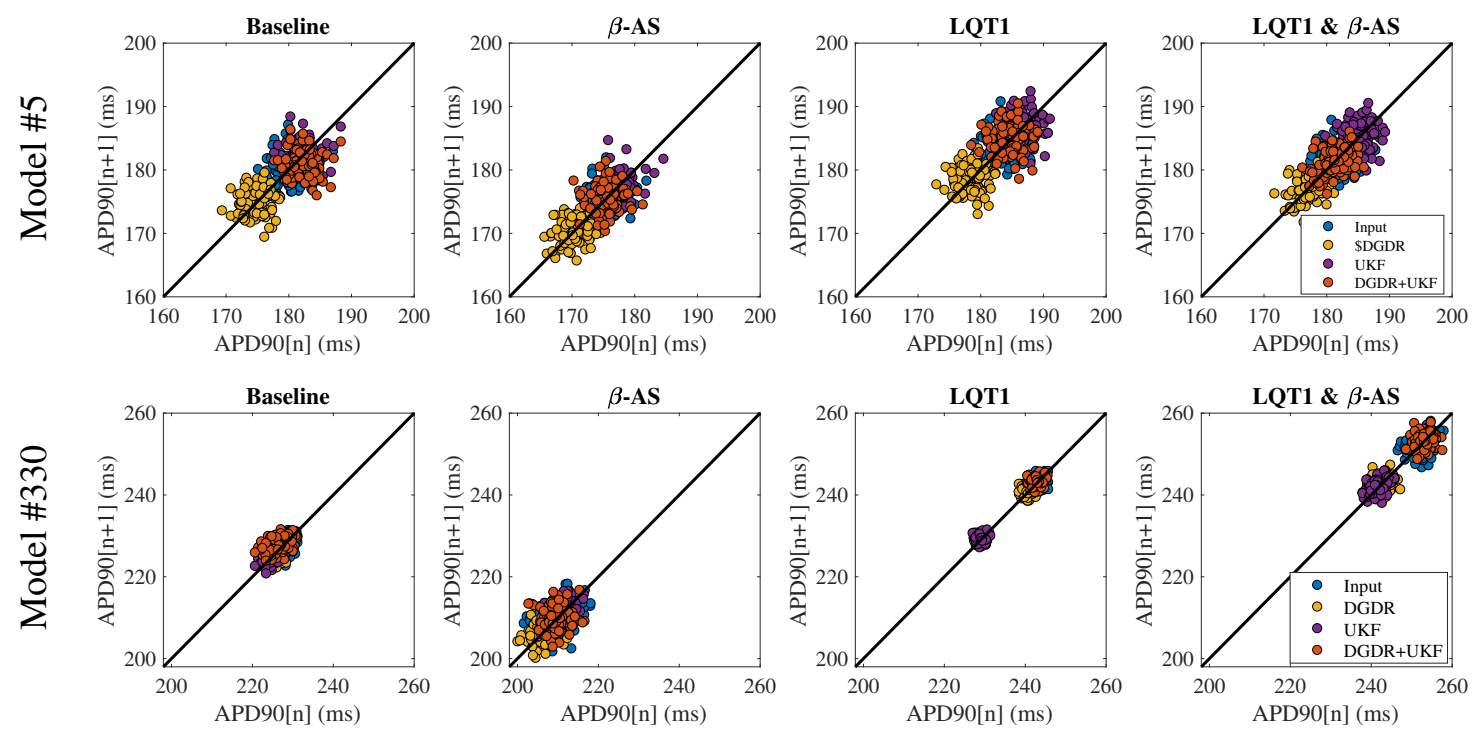

Figure 1. Poincaré plots of $\mathrm{APD}_{90}$, for two different virtual cells (models \#5 and \#330 in the population of Section 2.1), calculated from input voltage traces (blue) and from DGDR (yellow), UKF (purple) and DGDR-UKF (orange) estimates under four conditions: baseline, $\beta$-AS, LQT1 and LQT1 with $\beta$-AS.

The superior performance of DGDR-UKF illustrated in Fig. 1 for two particular cells were confirmed in the whole population of cells. Fig. 2 shows the probability density function of the error computed as the difference between mean APD (analogously for STV) from input and estimated AP traces. Those density functions are presented for two of the analyzed scenarios, namely $\beta$-AS and LQT1 with $\beta$-AS. From these results, it can be concluded that DGDR-UKF is able to more accurately represent the duration and beat-to-beat variability of AP repolarization as compared to individual DGDR and UKF methods.

It is interesting to highlight that DGDR-UKF was able to closely reproduce APD and STV in all models of the population independently of whether $\beta$-AS and LQT1 led to increased, decreased or preserved APD. This is relevant, particularly considering the high level of inter-individual variability in such responses, with APD changes ranging from $-86 \mathrm{~ms}$ to $12 \mathrm{~ms}$ in response to $\beta$-AS under control conditions, from $1 \mathrm{~ms}$ to $196 \mathrm{~ms}$ under LQT1 as compared to control and from $-24 \mathrm{~ms}$ to $178 \mathrm{~ms}$ under LQT1 with $\beta$-AS as compared to control without $\beta$-AS.

\subsection{Proarrhythmic Repolarization Alter- nans in LQT1}

To further assess the performance of the analyzed estimation methods, their capability to reproduce arrhythmogenic adrenergic-induced APD alternans in LQT1 was tested. Fig. 3 shows input and estimated AP traces for a virtual cell in the model population under pacing at a frequency of $5.3 \mathrm{~Hz}$. As can be observed from the figure, APD alternans ocurred at this frequency. For that cell,
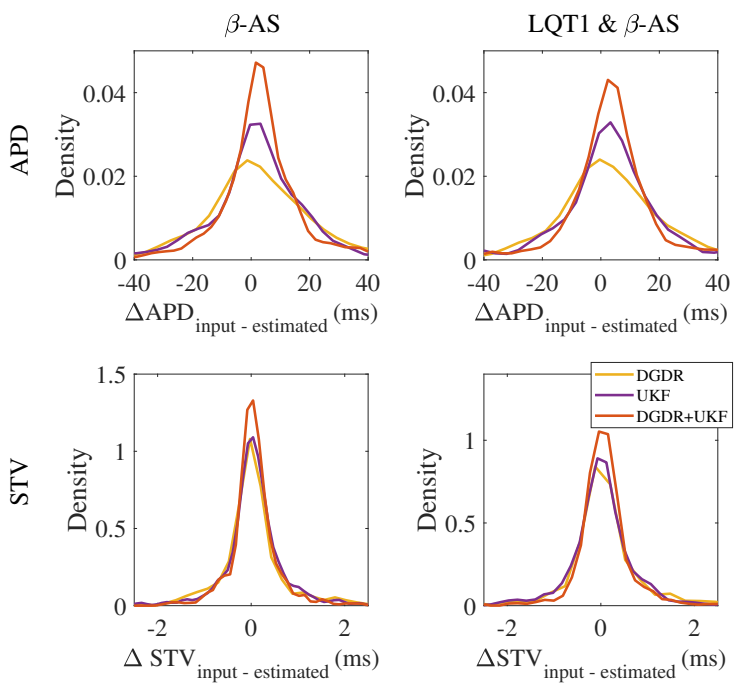

Figure 2. Probability density function of the error between input and estimated APD (top) and STV (bottom) over the population of models described in Section 2, for two different scenarios, $\beta$-AS (left) and LQT1 with $\beta$-AS (right).

APD alternans were observed for all frequencies spanning from 4.5 to $5.5 \mathrm{~Hz}$. This phenomenon was well reproduced by DGDR-UKF estimation, with the alternating pattern of the APD time series and of the whole AP morphology for the input and estimated traces being very similar. On the other hand, for the same virtual cell of Fig. 3, individual DGDR and UKF methods did not produce alternans for any tested pacing frequency from 2 to $10 \mathrm{~Hz}$. This result emphasizes the need for accurate identification methods, as small errors in the estimation of ionic current conductances and/or phosphorylation levels can lead to AP mod- 

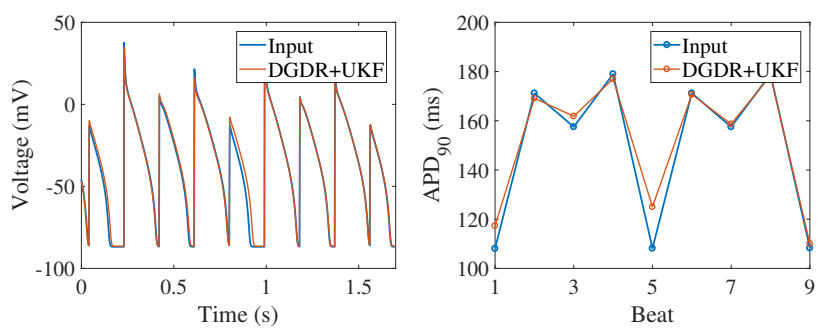

Figure 3. Left: Input (blue) and estimated (orange) AP traces by DGDR for a virtual cell of the model population described in Section 2, showing adrenergic-induced APD alternans under LQT1 when paced at 5.3 Hz. Right: Corresponding APD time series.

els not able to reproduce relevant proarrhythmic phenomena observed in the original input data, like repolarization alternans, or to reproduce them but in a different range of pacing frequencies, as observed for other cells of our experimentally-calibrated population. It is interesting to note that, although the alternans pattern shown in Fig. 3 is not the typical 1:1 short-long pattern with all short (respectively, long) APDs having similar values, the presented APD time series shows differences between consecutive beats that are above $14 \mathrm{~ms}$ in all cases.

\section{Conclusion}

A method combining the dimension reduction DGDR method and the nonlinear UKF reliably identifies an underlying AP model for any input ventricular voltage trace, allowing in silico characterization of repolarization duration and variability in the LQT1 syndrome. Importantly, proarrhythmic phenomena occurring in LQT1 under $\beta$-AS, like APD alternans, are well captured by the proposed method. This research sets the basis for investigations aimed at disentangling the mechanisms underlying arrhythmias in the LQT1 syndrome in response to $\beta$-AS.

\section{Acknowledgments}

This work was supported by projects ERC-StG 638284 (ERC), PID2019-105674RB-I00 and PID2019104881RB-I00 (MICINN), project LMP124-18, reference group T39-20R, a personal PhD grant to D.A (Aragón Government cofunded by FEDER 2014-2020 "Building Europe from Aragón") and grants by Campus Iberus through Erasmus+ program, Fundación CAI-Ibercaja and INRIA Foundations. Computations were performed using ICTS NANBIOSIS (HPC Unit at University of Zaragoza).

\section{References}

[1] Schwartz PJ, Priori SG, Spazzolini C, Moss AJ, Vincent GM, Napolitano C, Denjoy I, Guicheney P, Breithardt G, Keating MT, Towbin JA, Beggs AH, Brink P, Wilde AAM,
Toivonen L, Zareba W, Robinson JL, Timothy KW, Corfield V, Wattanasirichaigoon D, Corbett C, Haverkamp W, Schulze-Bahr E, Lehmann MH, Schwartz K, Coumel P, Bloise R. Genotype-Phenotype Correlation in the Long-QT Syndrome. Circulation 2001;103(1):89-95. ISSN 00097322.

[2] Gallacher DJ, Van de Water A, van der Linde H, Hermans AN, Lu HR, Towart R, Volders PG. In vivo mechanisms precipitating Torsades de Pointes in a canine model of drug-induced long-QT1 syndrome. Cardiovas Res 2007; 76(2):247-256.

[3] Lombardi D, Raphel F. A greedy dimension reduction method for classification problems. HAL 2019; hal02280502.

[4] Julier SJ, Uhlmann JK. A new extension of the Kalman filter to Nonlinear systems. In AeroSense '97. Proc. of AeroSense: The 11th Int. Symp. on Aerospace/Defence Sensing. Simulation and Controls., 1997; 182-193.

[5] Sampedro-Puente DA, Fernandez-Bes J, Porter B, van Duijvenboden S, Taggart P, Pueyo E. Mechanisms Underlying Interactions Between Low-Frequency Oscillations and Beat-to-Beat Variability of Celullar Ventricular Repolarization in Response to Sympathetic Stimulation: Implications for Arrhythmogenesis. Front Physiol 2019;10:916.

[6] Sampedro-Puente DA, Raphel F, Fernandez-Bes J, Laguna P, Lombardi D, Pueyo E. Characterization of spatiotemporal cardiac action potential variability at baseline and under $\beta$-adrenergic stimulation by combined unscented kalman filter and double greedy dimension reduction. IEEE J Biomed Health Inform 2020;ISSN 2168-2194.

[7] O'Hara T, Virág L, Varró A, Rudy Y. Simulation of the undiseased human cardiac ventricular action potential: model formulation and experimental validation. PLoS Comput Biol 2011;7(5):e1002061. ISSN 1553-7358.

[8] Xie Y, Grandi E, Puglisi JL, Sato D, Bers DM. $\beta$-adrenergic stimulation activates early afterdepolarizations transiently via kinetic mismatch of PKA targets. J Mol Cell Cardiol 2013;58:153-161.

[9] Soltis AR, Saucerman JJ. Synergy between CaMKII substrates and $\beta$-adrenergic signaling in regulation of cardiac myocyte Ca2+ handling. Biophys J 2010;99(7):2038-2047.

[10] Pueyo E, Orini M, Rodríguez JF, Taggart P. Interactive effect of beta-adrenergic stimulation and mechanical stretch on low-frequency oscillations of ventricular action potential duration in humans. J Mol Cell Cardiol 2016;97:93-105.

[11] Sampedro-Puente DA, Fernandez-Bes J, Virág L, Varró A, Pueyo E. Data-driven identification of stochastic model parameters and state variables: Application to the study of cardiac beat-to-beat variability. IEEE J Biomed Health Inform 2020;24(3):693-704.

Address for correspondence:

David Adolfo Sampedro Puente

Universidad de Zaragoza, C/ Mariano Esquillor S/N.

Edif. I+D+i, Lab. 6.1.1, 50018, Zaragoza.

sampedro@unizar.es 